\title{
Omnipresence and Absolutism
}

\section{Arjmand SA*}

Shiraz Electronics Industries, Iran

*Corresponding author: Sayed Abolfazl Arjmand, Shiraz Electronics Industries, Khiaban Eslah Nejad, Kucheh 1, Aparteman Amir, Shiraz, Iran, Tel: +98 917317 7523;

\section{Short Communication}

Volume 1 Issue 1

Received Date: August 03, 2018

Published Date: August 22, 2018

DOI: $10.23880 /$ phij-16000103

Email: abarjm@yahoo.com

Keywords: Omnipresence; Concept; Relationists; Absolutism

When we are pointing to somewhere, in actual fact we are pointing to something. For example, we show a chair in our room and say 'here', or we show a star in the sky and say 'there'. The chair and the star are two objects that represent two locations. A line of sight can be drawn between us and the object that we are pointing to. This line indicates a direction. If there is no object in this direction, the line of sight extends to infinity and we cannot determine which place on this endless line we are pointing to. We need an object to mark a location in the space.

Now suppose that we are diving in a deep ocean and there is nothing around us except the unbounded water. Again we cannot locate any place even though we know that there is some object taking up all the space around us, that is, the water. We can point to an object only if it occupies a limited volume and if we are standing out of it. If an object occupies all the space, we cannot point to it and it cannot be used to refer to a particular location. The situation when there is no object is similar to when there is an object that has filled everywhere. If all the space is uniformly filled with something, it looks like there is nothing in the space.

Ocean, even if it contains pure water, is not perfectly uniform, because water has granular structure and microscopically its molecules are separate from each other and have gaps between them. Theoretically we can single out a certain molecule of water and use it as a reference to locate a certain place in the ocean. Perfect uniformity is when something has not granular structure and truly fills all points of the space. If all the space is filled with a perfectly uniform substance, this substance cannot be used to point to somewhere, the same situation as when there is nothing in the space.

Omnipresence is the presence of something everywhere. If something is omnipresent, it looks as if it is absent. An approximate example of omnipresence is the water in a great ocean. Another approximate example is the air surrounding us on the Earth. Air fills everywhere, but we do not see it anywhere and we cannot use it to refer to a particular point on the Earth.

Consider an 'empty' water bottle. We know that the bottle is not really empty, but it is full of air. Since air is present everywhere, it looks as if it is absent and we neglect its presence in the bottle. If we want to fill the bottle with water, we should let the air go out. But even a bottle full of water may appear to be empty. Suppose that a diver finds a bottle at the bottom of the ocean. He may have a close look to see if there is something in the bottle, but he finds 'nothing' inside. This time, although the bottle is full of water, it is described as empty again. In an ocean of water, we only pay attention to objects other than water.

The analogy of ocean and the concept of omnipresence can be applied to build a theory of space and time. At first we assume an omnipresent being, an approximation of which can be imagined as a great uniform ocean of water occupying everywhere. In this ocean there is no limited creature to refer to its location, and there is no change to refer to its time. Then, according to the theory of evolution, a tiny fish evolves out of the water. Here it is not important how this process happens. From then on, we have a certain place in the ocean to refer to, that is, where the fish locates. We also have a change in the ocean, before which we could see nothing and after which we see something. This change is a reference of time. 
Birth of a tiny creature in the ocean provides a reference point of space and time. The fish is an object, and its birth is an event. We observe many objects and events around us. Objects correspond to places and events correspond to times.

As another example, we can imagine ourselves in a dark environment where there are no objects and no events observable. Then a point of light, for example a star, appears in the darkness. The star is an object and its appearance is an event. From then on, we have a point in the space to refer to its position. Furthermore, we can differentiate between the situations when it was total darkness and now that we have a point of light. This change reveals a reference point of time.

Darkness, like emptiness, is conception caused by the limitations of our senses. Human eyes can sense only visible frequencies which are a small fraction of the electromagnetic spectrum. If we could see all the wavelengths, nowhere would be dark. But in an environment that our eyes receive a uniform steady light from all directions, we cannot see anything, the same as when we are in total darkness. Space and time are revealed to us only if we observe a non-uniform, changing environment.

There are two main theories about space and time: relational and absolute. Relationists believe that space and time are nothing in themselves, but relationships between the existents. If there is no object and no event, there is no space and no time. From their point of view, space and time are ideal concepts, not real ones. Absolutists, on the other hand, believe that space and time exist even if there is no object or event.

An outstanding figure of relationism was Leibniz, and his absolutist opponent was Newton. The debate between these two scientists and philosophers began in 17th century, and the struggle between the supporters of the two sides continues to present time. Here we apply the concept of omnipresence to defend absolutism.

To explain the relations between the objects and the space, Leibniz uses the analogy of family tree which is a diagram representing how the members of a family are related to each other. A member's place in the family is defined by their relationship to other members. Members are real things but relationships are ideal. If there is no member, there is no relationship. Leibniz claims that space and time are similar to family relations, that is, they are ideal relations between real objects and events. We see that in this analogy, the relationists need more than one existent to establish a relationship. If there is just one object or one event, there is no relation. But in our analogy of the ocean, if only one fish is born, a point of space and time is revealed. We just need one single object to have a perception of space, and just one single event to have a perception of time. In a family tree, we have no relationship if there is only one member, but in an ocean, a place is recognizable if there is only one fish.

Relationist view cannot distinguish between two completely different situations: when there is nothing at all, and when there is a uniform, unbounded, changeless substance that has filled everywhere. The existence of such an omnipresent being can be concluded by observing the creatures. We see that the creatures 'come into the world' and 'go out of the world.' They have lifetimes. If we believe that nothing comes out of nothing and nothing destroys into nothing, then there must be something in the background of the apparent world that the creatures evolve out of it and return back to it. The creatures can be the fishes swimming in the ocean with lifetimes of few years, or the celestial bodies 'swimming' in the sky with lifetimes of billions of years. Every creature at each location reveals a point of space and at each state reveals a point of time. Relationists neglect the background of the world and pay attention only to the foreground objects and events.

In an ocean, the water is the background substance while the creatures evolved from the water are the foreground objects. These objects are nothing but transformations of what previously existed. A fish is a transformation of the sea water and all its contents. When a creature is born in the sea, we have a reference point of space and time. When it dies and returns back to the sea, it continues to exist in a new form that cannot be distinguished from the background. We have a perception of space and time when we observe the discrete creatures in the foreground, but we know that the existence of the creatures continues after their death and returning back to the sea, even though we cannot observe them in their new form. Space and time continue to exist after the creatures have merged with their original substance and we apparently see an empty ocean.

Relationists consider the world to be a composition of many things, that is, many objects and many events. In his famous correspondence with Clarke, Leibniz says that space is an order of coexistences, that is, an order of things that exist at the same time. In a similar way, time is an order of successions, that is, an order of things succeeding each other. From this point of view, what we call space and time are two ways that the objects and events are ordered relating to one another. No object and no event mean no space and no time. But the multiple bounded beings that the relationists use to define space 


\section{Philosophy International Journal}

and time have evolved out of a single boundless being that exist everywhere all the time. Things have evolved out of one omnipresent thing. Apparently we observe many discrete creatures in the foreground but logically there is a single continuous being in the background.

While the creatures are bounded and have limited lifetimes, the ocean itself is boundless and timeless. Before the creatures' birth, the time passes in what we apparently see as an empty ocean which is actually full of water. Similarly, when the ocean is empty of creatures, the space exists in the ocean and all the locations are filled with water.

If we can perceive space and time in an ocean full of creatures, they also exist in an apparently empty ocean. This is absolutism. Space and time exist independent of our observation of the objects and the events. 